\author{
International Journal of Mathematical Analysis \\ Vol. 9, 2015, no. 1, 1 - 14 \\ HIKARI Ltd, www.m-hikari.com \\ http://dx.doi.org/10.12988/ijma.2015.411352
}

\title{
The Application Domain of Cesàro Matrix on Some Sequence Spaces of Fuzzy Numbers
}

\author{
Zarife Zararsız and Mehmet Şengönül \\ Nevşehir Hacı Bektaş Veli University \\ Department of Mathematics \\ 2000 Evler Mah. 50300 \\ Nevşehir, Turkey
}

Copyright (c) 2014 Zarife Zararsız and Mehmet Şengönül. This is an open access article distributed under the Creative Commons Attribution License, which permits unrestricted use, distribution, and reproduction in any medium, provided the original work is properly cited.

\begin{abstract}
The main purpose of this paper is to construct the Cesàro sequence spaces of triangular fuzzy numbers $\left[\ell_{\infty}\left(F_{t}\right)\right]_{\mathscr{C}},\left[c\left(F_{t}\right)\right]_{\mathscr{C}}$ and $\left[c_{0}\left(F_{t}\right)\right]_{\mathscr{C}}$ consisting of all sequences $u=\left(u_{k}\right)$ such that $\mathscr{C} u$ is in the spaces $\ell_{\infty}(F), c(F)$ and $c_{0}(F)$, respectively. It is proved that the Cesàro sequence spaces of triangular fuzzy numbers $\left[\ell_{\infty}\left(F_{t}\right)\right]_{\mathscr{C}},\left[c\left(F_{t}\right)\right]_{\mathscr{C}}$ and $\left[c_{0}\left(F_{t}\right)\right]_{\mathscr{C}}$ are linearly isomorphic to the spaces $\ell_{\infty}(F), c(F)$ and $c_{0}(F)$, respectively. Additionally, the $\alpha(r)-, \beta(r)$ - and $\gamma(r)$ - real duals of the spaces $\left[\ell_{\infty}\left(F_{t}\right)\right]_{\mathscr{C}},\left[c\left(F_{t}\right)\right]_{\mathscr{C}}$ and $\left[c_{0}\left(F_{t}\right)\right]_{\mathscr{C}}$ have been computed. Finally, the classes of $\left(\left[c\left(F_{t}\right)\right]_{\mathscr{C}}: \mu(F)\right)$ and $\left(\mu(F):\left[c\left(F_{t}\right)\right]_{\mathscr{C}}\right)$, are characterized, where $\mu(F)$ is any sequence space.
\end{abstract}

Mathematics Subject Classification: 03E72; 46A45; $40 \mathrm{C} 05$

Keywords: fuzzy numbers, Cesàro matrix, matrix transformations, real dual

\section{Introduction}

The concept of fuzzy sets and fuzzy set operations were first introduced by Zadeh [12]. After his innovation many authors have studied various aspects 
of the fuzzy set theory and its applications, such as fuzzy topological spaces, similarity relations and fuzzy mathematical programming. In the presented paper, we introduce the sequence spaces of triangular fuzzy numbers represented as follows $\left[\ell_{\infty}\left(F_{t}\right)\right]_{\mathscr{C}},\left[c\left(F_{t}\right)\right]_{\mathscr{C}}$ and $\left[c_{0}\left(F_{t}\right)\right]_{\mathscr{C}}$. Additionally, we redefine fuzzy identity elements according to addition and multiplication for constructing an algebraic structure. It will not be right to regard this article as a copy of classic summability theory because both a big generalization and definitions of fuzzy zero are presented in this article. Therefore, the readers are advised to take these into consideration while reading the article, and using this definition of fuzzy zero, we have solved some equation in the form $x+a=b$, where $a$ and $b$ are fuzzy number. Additionally, for the convenience in this article, we have used the same terminology in [9]. We would like to express that the rest of our paper is organized, as follows:

In Section 2, some basic definitions and theorems related to the fuzzy numbers are given. In Section 3, we have introduced Cesàro sequence space of triangular fuzzy numbers and proved some inclusion relations on these sequence spaces. In Section 3, it is also established that the sequence spaces of triangular fuzzy numbers showed by $\left[\ell_{\infty}\left(F_{t}\right)\right]_{\mathscr{C}},\left[c\left(F_{t}\right)\right]_{\mathscr{C}}$ and $\left[c_{0}\left(F_{t}\right)\right]_{\mathscr{C}}$ are linearly isomorphic to the spaces $\ell_{\infty}\left(F_{t}\right), c\left(F_{t}\right)$ and $c_{0}\left(F_{t}\right)$, respectively. In Section 3 , it is proved that the spaces $\left[\ell_{\infty}\left(F_{t}\right)\right]_{\mathscr{C}},\left[c\left(F_{t}\right)\right]_{\mathscr{C}}$ and $\left[c_{0}\left(F_{t}\right)\right]_{\mathscr{C}}$ are complete with the module defined by $\|u\|=\sup _{k} \max _{i=1,2,3}\left\{\left|\left(\mathscr{C} u_{k}^{(i)}\right)\right|\right\}$. Section 4 is devoted to the calculation of the $\alpha(r)$-, $\beta(r)$ - and $\gamma(r)$ - duals of the spaces $\left[c\left(F_{t}\right)\right]_{\mathscr{C}}$ and $\left[c_{0}\left(F_{t}\right)\right]_{\mathscr{C}}$. In Section 5, some classes of matrix transformations from the space $\left[c\left(F_{t}\right)\right]_{\mathscr{C}}$ and $\left[c_{0}\left(F_{t}\right)\right]_{\mathscr{C}}$ to $\mu(F)$ and from $\mu(F)$ to $\left[c\left(F_{t}\right)\right]_{\mathscr{C}}$ and $\left[c_{0}\left(F_{t}\right)\right]_{\mathscr{C}}$ by employing the suitable relations between the related matrix classes are characterized, respectively, where $\mu(F)$ is any sequence space.

\section{Preliminary Notes}

Let's suppose that $\mathbb{N}, \mathbb{R}$ and $E_{i}$ be the set of all positive integers, all real numbers and all bounded and closed intervals on the real line $\mathbb{R}$, i.e., $E_{i}=$ $\left\{a=\left[a^{-}, a^{+}\right]: a^{-} \leq x \leq a^{+}, a^{-}\right.$and $\left.a^{+} \in \mathbb{R}\right\}$, respectively. It can easily be seen that $d(a, b)=\max \left\{\left|a^{-}-b^{-}\right|,\left|a^{+}-b^{+}\right|\right\}$defines a metric on $E_{i}$ for $a, b \in E_{i}$ and the pair $\left(E_{i}, d\right)$ is a complete metric space [5]. Let $X$ be a nonempty set. According to Zadeh a fuzzy subset of $X$ is a nonempty subset $\{(x, u(x)): x \in X\}$ of $X \times[0,1]$ for some function $u: X \rightarrow[0,1]$, [6]. Consider a function $u: \mathbb{R} \rightarrow[0,1]$ as a subset of a nonempty base space $\mathbb{R}$ and denote the family of all such functions or fuzzy sets by $E$. Let us suppose that the function $u$ satisfies the following properties:

(5) $\mathrm{u}$ is normal, i.e., there exists an $x_{0} \in \mathbb{R}$ such that $u\left(x_{0}\right)=1$,

(6) $\mathrm{u}$ is fuzzy convex, $u[\mu x+(1-\mu) y] \geq \min \{u(x), u(y)\}$ 
(7) $\mathrm{u}$ is upper semi-continuous,

(8) The closure of $\{x \in \mathbb{R}: u(x)>0\}$, denoted by $u^{0}$, is compact.

Then the function $u$ is called a fuzzy number, [6]. The properties (5)-(8) imply that for each $\alpha \in[0,1]$, the $\alpha$ - cut set of the fuzzy number $u$ defined by $u(\alpha)=\{x \in \mathbb{R}: u(x) \geq \alpha\}$ is in $E_{i}$. That is the equality $u(\alpha)=\left[u^{-}(\alpha), u^{+}(\alpha)\right]$ holds for each $\alpha \in[0,1]$. We denote the set of all fuzzy numbers by $F$ and write $\ell_{\infty}, c, c_{0}$ and $\ell_{p}$ for the classical sequence spaces of all bounded, convergent, null and absolutely $p$ - summable sequences. Also by $b s$ and $c s$, we represent the spaces of all bounded and convergent series, respectively. Sometimes, the representation of fuzzy numbers with $\alpha$ - cut sets induce errors according to algebraic operations. For example, if $u$ is any fuzzy number then $u-u=$ $\left[u^{-}(\alpha), u^{+}(\alpha)\right]-\left[u^{-}(\alpha), u^{+}(\alpha)\right]=\left[u^{-}(\alpha)-u^{+}(\alpha), u^{+}(\alpha)-u^{-}(\alpha)\right]$ is not equal to zero.

In this study, by following Şengönül [9] we have used another type representation of a fuzzy number to avoid the same algebraic errors as used in [3], $[7]$.

Furthermore, we know that shape similarity of the membership functions does not reflect the conception of itself, but it will be used for examining the context of the membership functions. However, not that many applications are overly sensitive to variations in the shape. In such cases, it is convenient to use a simple shape, such as the triangular shape of membership function.

For example, let us consider any triangular fuzzy number $u$, as in the following:

If the function $u(x)=\left\{\begin{array}{cl}\frac{x-u^{1}}{u^{2}-u^{1}}, & x \in\left[u^{1}, u^{2}\right] \\ \frac{u^{3}-x}{u^{3}-u^{2}}, & x \in\left[u^{2}, u^{3}\right] \text { is the membership function } \\ 0, & \text { otherwise }\end{array}\right.$ of the triangular fuzzy number $u$ then $u$ can be represented with the notation $u=\left(u^{1}, u^{2}: 1, u^{3}\right)$. Clearly this representation is unique for $u(x)$ and on the contrary there is an unique $u(x)$ for every $u$. After here, the triangular fuzzy number will be denoted shortly $u=u^{(1,2: 1,3)}$ and the set of all triangular fuzzy numbers denoted by $F_{t}$, that is

$$
F_{t}=\left\{u=u^{(1,2: 1,3)}: u^{1} \leq u^{2: 1} \leq u^{3} \text { and } u^{1}, u^{2: 1}, u^{3} \in \mathbb{R}\right\}
$$

The $u^{1}, u^{2: 1}$ and $u^{3}$ are called first, middle and end points of triangular fuzzy number, respectively. The notation $u^{2}: 1$ means that the height of the fuzzy number $u$ is 1 at the point $u^{2}$. There are two important and different structure on any of the set. These are algebraic and topological structures.

The algebraic structure of $F$ is defined as follows:

Let $u, v \in F$ and $\lambda \in \mathbb{R}$. The operations addition and scalar multiplication is defined on $F$ as follows:

$$
u+v=u^{(1,2,3)}+v^{(1,2,3)}=\left(u^{1}+v^{1}, u^{2}+v^{2}, u^{3}+v^{3}\right)=w^{(1,2,3)}=w
$$




$$
\lambda u^{(1,2,3)}=\left\{\begin{array}{ll}
\left(\lambda u^{1}, \lambda u^{2}, \lambda u^{3}\right) & \text { for } \lambda \geq 0 \\
\left(\lambda u^{3}, \lambda u^{2}, \lambda u^{1}\right) & \text { for } \lambda<0
\end{array} .\right.
$$

It is in fact that it is necessary to make some assumptions on the set of fuzzy numbers used in the construction of an algebraic structure. Due to this reason we will use fuzzy identity elements as defined by Şengönül [9], according to addition and multiplication as in the following. Let suppose that $u^{1} \leq 0$, $u^{2}=0$ and $u^{3} \geq 0$. If we accept that any fuzzy number

$$
\overline{0}=\left(u^{1}, 0, u^{3}\right)
$$

is a fuzzy zero then the difference $u^{(1,2,3)}-u^{(1,2,3)}=u^{(1-3,2-2,3-1)}=\left(u^{1}-\right.$ $\left.u^{3}, 0, u^{3}-u^{1}\right)=\overline{0}$ is acceptable for representing fuzzy zero for us. Therefore, we can say that, the inverse of the fuzzy number $u^{(1,2,3)}$ is $-u^{(1,2,3)}=$ $\left(-u^{3},-u^{2},-u^{1}\right)$, according to addition and $-u^{(1,2,3)}$ determines a fuzzy number. With this idea, we can solve equations in the form $x^{(1,2,3)}+u^{(1,2,3)}=\overline{0}$ which may be given by an inexact data. The "fuzzy number a" can be denoted by the notation $\left(u^{1}, a, u^{3}\right)$ and $K=\max \left\{\left|u^{1}-a\right|,\left|a-u^{3}\right|\right\}$. It should be that $K<1$. Theoretically, the condition $K<1$ is not necessary but has to be in practical. For example, the "approximately 5 " can be taken as $\overline{5}=(-4,5,15)$ but in the applications, generally, "approximately 5 " taken as $\overline{5}=(5-t, 5,5+t),(0<t<1)$. This choice is more accurate than $\overline{5}=(-4,5,15)$. We know that, generally, in the practical applications, the spread of fuzziness is not very large. Furthermore, it must be emphasized that a fuzzy number is determined according to specific processes and this fuzzy number $u$ may not in the same sense in the another specific system. Let $A$ and $B$ be two different specific system. If $u$ is approximately 5 for the specific system $A$ then the approximately 5 will not be in the same manner for another system $B$. So, algebraic properties of the systems $A$ and $B$ are different. This can be explained as follows: Let suppose that the spread left and right fuzziness of every fuzzy number $u$ be equal to $a$ in the system $A$. Then fuzzy zero for system $A$ is $(-2 t, 0,2 t)$ and this fuzzy zero is unique for system $A$. It is a fact that, the set $F_{t}$ is not a linear space according to algebraic operations in sense of (2) and (3).

Şengönül [9] has constructed a topology on $F_{t}$ by using the metric $\bar{d}$ : $F_{t} \times F_{t} \longrightarrow \mathbb{R}$ defined as follows, $\bar{d}\left(u^{(1,2,3)}, v^{(1,2,3)}\right):=\max _{i=1,2,3}\left\{\left|u^{i}-v^{i}\right|\right\}$. We can easily show that the set $F_{t}$ is a complete metric space with the metric $\bar{d}$. The function $f: \mathbb{N} \rightarrow F_{t}, k \rightarrow f(k)=u_{k}^{(1,2,3)}$ is called a sequence of fuzzy numbers and is represented by $\left(u_{k}\right)=\left(u_{k}^{(1,2,3)}\right)$, [4]. Let us denote the set of all sequences of fuzzy numbers by $w\left(F_{t}\right)$, that is, $w\left(F_{t}\right):=\left\{u=\left(u_{k}^{(1,2,3)}\right): u\right.$ : $\left.\mathbb{N} \rightarrow F_{t}, u(k)=u_{k}^{(1,2,3)}\right\}$, where $u_{k}^{1} \leq u_{k}^{2} \leq u_{k}^{3}$ and $u_{k}^{1}, u_{k}^{2}, u_{k}^{3} \in \mathbb{R}$ for all $k \in \mathbb{N}$, $[13]$.

If degree of membership at $u_{k}^{2}$ is equal to 1 then $\left(u_{k}\right)$ is a sequence of fuzzy numbers, if it is not equal to 1 then the sequence $\left(u_{k}\right)$ is only a sequence of the 
fuzzy sets. Each subspace of $w\left(F_{t}\right)$ is called a sequence space of triangular fuzzy numbers. We define the classical sets $\ell_{\infty}\left(F_{t}\right), c\left(F_{t}\right), c_{0}\left(F_{t}\right)$ and $\ell_{p}\left(F_{t}\right)$ consisting of the bounded, convergent, null and absolutely $p$ - summable sequences of fuzzy numbers, respectively, it means that,

$$
\begin{aligned}
& \ell_{\infty}\left(F_{t}\right)=\left\{u=\left(u_{k}^{(1,2,3)}\right) \in w\left(F_{t}\right): \sup _{k \in \mathbb{N}} \bar{d}\left(u_{k}^{(1,2,3)}, \overline{0}\right)<\infty\right\}, \\
& c\left(F_{t}\right)=\left\{u=\left(u_{k}^{(1,2,3)}\right) \in w\left(F_{t}\right): \lim _{k} \bar{d}\left(u_{k}^{(1,2,3)}, u_{0}^{(1,2,3)}\right)=0, \quad u_{0} \in F_{t}\right\}, \\
& c_{0}\left(F_{t}\right)=\left\{u=\left(u_{k}^{(1,2,3)}\right) \in w\left(F_{t}\right): \lim _{k} \bar{d}\left(u_{k}^{(1,2,3)}, \overline{0}\right)=0\right\}, \\
& \ell_{p}\left(F_{t}\right)=\left\{u=\left(u_{k}^{(1,2,3)}\right) \in w\left(F_{t}\right): \sum_{k} \bar{d}\left(u_{k}^{(1,2,3)}, \overline{0}\right)^{p}<\infty, 1 \leq p<\infty\right\} .
\end{aligned}
$$

We should emphasize here that $\ell_{\infty}\left(F_{t}\right), c\left(F_{t}\right), c_{0}\left(F_{t}\right)$ and $\ell_{p}\left(F_{t}\right)$ can be reduced to the classical spaces of real numbers $\ell_{\infty}, c, c_{0}$ and $\ell_{p}$, respectively in the case $\left(u_{k}^{(1,2,3)}\right)=\left(u_{k}^{(1,1,1)}\right)$, where $u_{k}^{1} \in \mathbb{R}$ and $u_{k}^{(1,2,3)} \in F_{t}$. So, the properties and results related to the spaces of $\ell_{\infty}\left(F_{t}\right), c\left(F_{t}\right), c_{0}\left(F_{t}\right)$ and $\ell_{p}\left(F_{t}\right)$ are more general and useful than the corresponding consequences of the spaces $\ell_{\infty}, c, c_{0}$ and $\ell_{p}$, respectively.

Definition 2.1 Let $\lambda\left(F_{t}\right) \subset w\left(F_{t}\right), \theta$ is identity element of $\lambda\left(F_{t}\right)$ according to addition and algebraic operations on $\lambda\left(F_{t}\right)$ sense of $(2)$ and $(3)$. The function $\|\|:. \lambda\left(F_{t}\right) \rightarrow \mathbb{R}$ is called module on the set $\lambda\left(F_{t}\right)$ if the following properties hold: (15) $\|u\|=0 \Leftrightarrow u=\theta,(16)\|\alpha u\|=|\alpha|\|u\|,(17)\|u+v\| \leq\|u\|+\|v\|$.

If the function $\|\|:. \lambda\left(F_{t}\right) \rightarrow \mathbb{R}$ satisfy (15)-(17) then $\lambda\left(F_{t}\right)$ is called the module of sequence space of the fuzzy numbers. If $\lambda\left(F_{t}\right)$ is complete with respect to the module $\|$.$\| then \lambda\left(F_{t}\right)$ is called complete module sequence space of the fuzzy numbers. Because of $\lambda\left(F_{t}\right)$ is not linear space, in the usual sense, the function $\|\cdot\|$ is called a modul by us.

Lemma $2.2[9]$ The sets $c\left(F_{t}\right), c_{0}\left(F_{t}\right)$ and $\ell_{\infty}\left(F_{t}\right)$ are complete module sequence spaces with the module defined $\|u\|=\sup _{k} \max _{i=1,2,3}\left\{\left|u_{k}^{(i)}\right|\right\}$, where $u$ is in the any sets of $\left\{c\left(F_{t}\right), c_{0}\left(F_{t}\right), \ell_{\infty}\left(F_{t}\right)\right\}$.

Now, let $\lambda(F)$ and $\mu(F)$ be two spaces of fuzzy valued sequences and $\mathscr{A}=$ $\left(a_{n k}\right)$ be an infinite matrix of positive real numbers $a_{n k}$, where $n, k \in \mathbb{N}$. Then, we say that $\mathscr{A}$ defines a real- matrix mapping from $\lambda(F)$ to $\mu(F)$ and we denote it by writing $\mathscr{A}: \lambda(F) \rightarrow \mu(F)$, if for every sequence $u=\left(u_{k}^{(1,2,3)}\right) \in \lambda(F)$ the sequence $\mathscr{A} u=\left\{\left(\mathscr{A} u^{(1,2,3)}\right)_{n}\right\}$, is in $\mu(F)$ where

$$
\left(\mathscr{A} u^{(1,2,3)}\right)_{n}=\sum_{k} a_{n k}^{(1,1,1)} u_{k}^{(1,2,3)}=\left(\sum_{k} a_{n k}^{(1,1,1)} u_{k}^{1}, \sum_{k} a_{n k}^{(1,1,1)} u_{k}^{2}, \sum_{k} a_{n k}^{(1,1,1)} u_{k}^{3}\right) .
$$


By $(\lambda(F): \mu(F))$, we denote the class of matrices $\mathscr{A}$ such that $\mathscr{A}: \lambda(F) \rightarrow$ $\mu(F)$. Thus, $\mathscr{A} \in(\lambda(F): \mu(F))$ if and only if the series on the right side of (5) are convergent for each $n \in \mathbb{N}$ and every $u \in \lambda(F)$, and we have $\mathscr{A} u=$ $\left\{\left(\mathscr{A} u^{(1,2,3)}\right)_{n}\right\}_{n \in \mathbb{N}} \in \mu(F)$ for all $u \in \lambda(F)$.

Let the infinite matrix $\mathscr{C}=\left(c_{n k}\right)$ of positive real numbers be the Cesàro matrix of one order, that is,

$$
c_{n k}=\left\{\begin{array}{cll}
\frac{1}{n+1} & , \quad 0 \leq k \leq n \\
0 & , \quad \text { otherwise }
\end{array} ;(n, \quad k \in \mathbb{N}) .\right.
$$

Definition 2.3 Let $\lambda(F)$ be a sequence space of fuzzy numbers. Then, the set $[\lambda(F)]_{\mathscr{A}}$ of sequences of fuzzy numbers defined as follows, is called the fuzzy domain of an infinite matrix $\mathscr{A}$ in $\lambda(F)$,

$$
[\lambda(F)]_{\mathscr{A}}:=\left\{u=\left(u_{k}^{(1,2,3)}\right) \in w(F): \mathscr{A} u^{(1,2,3)} \in \lambda(F)\right\} .
$$

In special case, $[c(F)]_{\mathscr{A}}$ is called fuzzy convergence domain of the matrix $\mathscr{A}$.

\section{The Cesàro sequence spaces $\left[\ell_{\infty}\left(F_{t}\right)\right]_{\mathscr{C}},\left[c\left(F_{t}\right)\right]_{\mathscr{C}}$ and $\left[c_{0}\left(F_{t}\right)\right]_{\mathscr{C}}$.}

The $\mathscr{C}$ - transform of the sequence $\left(u_{k}\right)=((-2,-1,0),(0,1,2), \ldots,(-2,-1,0)$, $(0,1,2), \ldots)$ of fuzzy numbers is equal to $((-1,0,1))$ which means we can summable a divergent sequence of fuzzy numbers with this way. From here, we realize that, any limitation method can transform sequences of fuzzy numbers into set of all sequence of fuzzy numbers. Thus, we wish to introduce the $\left[\ell_{\infty}\left(F_{t}\right)\right]_{\mathscr{C}},\left[c\left(F_{t}\right)\right]_{\mathscr{C}}$ and $\left[c_{0}\left(F_{t}\right)\right]_{\mathscr{C}}$ spaces, as the set of all sequences such that $\mathscr{C}$ - transforms of them are in the spaces $\ell_{\infty}\left(F_{t}\right), c\left(F_{t}\right)$ and $c_{0}\left(F_{t}\right)$, respectively, in the meaning of (6), that is

$$
\begin{gathered}
{\left[\ell_{\infty}\left(F_{t}\right)\right]_{\mathscr{C}}=\left\{\left(u_{k}^{(1,2,3)}\right) \in w\left(F_{t}\right): \mathscr{C} u^{(1,2,3)} \in \ell_{\infty}\left(F_{t}\right)\right\}} \\
{\left[c\left(F_{t}\right)\right]_{\mathscr{C}}=\left\{\left(u_{k}^{(1,2,3)}\right) \in w\left(F_{t}\right): \mathscr{C} u^{(1,2,3)} \in c\left(F_{t}\right)\right\}}
\end{gathered}
$$

and

$$
\left[c_{0}\left(F_{t}\right)\right]_{\mathscr{C}}=\left\{\left(u_{k}^{(1,2,3)}\right) \in w\left(F_{t}\right): \mathscr{C} u^{(1,2,3)} \in c_{0}\left(F_{t}\right)\right\} .
$$

We should emphasize here that the sequence spaces $\left[\ell_{\infty}\left(F_{t}\right)\right]_{\mathscr{C}},\left[c\left(F_{t}\right)\right]_{\mathscr{C}}$ and $\left[c_{0}\left(F_{t}\right)\right]_{\mathscr{C}}$ of triangular fuzzy numbers can be reduced to the sets $\tilde{\ell}_{\infty}, \tilde{c}$ and $\tilde{c_{0}}$ 
respectively for the case $u_{k}^{(1,2,3)}=u_{k}^{(1,1,1)}$ for every $k \in \mathbb{N}$, where the sets $\tilde{\ell}_{\infty}, \tilde{c}$ and $\tilde{c_{0}}$ are defined in [8]. So, the properties and results related to sequence spaces of $\left[\ell_{\infty}\left(F_{t}\right)\right]_{\mathscr{C}},\left[c\left(F_{t}\right)\right]_{\mathscr{C}}$ and $\left[c_{0}\left(F_{t}\right)\right]_{\mathscr{C}}$ are more general and more extensive than the corresponding consequences of the spaces $\tilde{\ell}_{\infty}, \tilde{c}$ and $\tilde{c_{0}}$, respectively.

Define the sequence of triangular fuzzy numbers $v=\left(v_{n}^{(1,2,3)}\right)$ which will be frequently used, as the $\mathscr{C}$ - transform of a sequence of fuzzy numbers $u=$ $\left(u_{k}^{(1,2,3)}\right)$, that is,

$$
v_{n}^{(1,2,3)}=\frac{1}{n} \sum_{k=1}^{n} u_{k}^{(1,2,3)}
$$

where $k, n \in \mathbb{N}$. As we said above in (4), if we assume that each fuzzy number in the form $\left(u^{1}, 0, u^{3}\right)$ which is a fuzzy zero then $u_{k}^{(1,2,3)}$ can be left alone in the equality (10). This assumption is important for our idea, which is being used frequently in this paper.

Theorem 3.1 The sequence spaces $\left[\ell_{\infty}\left(F_{t}\right)\right]_{\mathscr{C}},\left[c\left(F_{t}\right)\right]_{\mathscr{C}}$ and $\left[c_{0}\left(F_{t}\right)\right]_{\mathscr{C}}$ are linearly isomorphic to the spaces $\ell_{\infty}\left(F_{t}\right), c\left(F_{t}\right)$ and $c_{0}\left(F_{t}\right)$, respectively.

Proof: Since the others can be similarly proved, we consider only case $\left[\ell_{\infty}\left(F_{t}\right)\right]_{\mathscr{C}} \cong \ell_{\infty}\left(F_{t}\right)$. To prove this, we should show the existence of a linear bijection between the spaces $\left[\ell_{\infty}\left(F_{t}\right)\right]_{\mathscr{C}}$ and $\ell_{\infty}\left(F_{t}\right)$. Consider the transformation defined $T$, with the notation of $(10)$, from $\left[\ell_{\infty}\left(F_{t}\right)\right]_{\mathscr{C}}$ to $\ell_{\infty}\left(F_{t}\right)$ by $u \mapsto v=T u=\frac{1}{n} \sum_{k=1}^{n} u_{k}^{(1,2,3)}$. The equality $T(u+w)=T u+T w$, where $u, w \in\left[\ell_{\infty}\left(F_{t}\right)\right]_{\mathscr{C}}$, is clear. Let us suppose that $a \geq 0$ then,

$$
T(a u)=T\left(a u_{k}^{(1,2,3)}\right)=\frac{1}{n} \sum_{k=1}^{n} a u_{k}^{(1,2,3)}=a \frac{1}{n} \sum_{k=1}^{n} u_{k}^{(1,2,3)}=a T u .
$$

If $a<0$ then,

$$
T(a u)=T\left(a u_{k}^{(1,2,3)}\right)=\frac{1}{n} \sum_{k=1}^{n} a u_{k}^{(3,2,1)}=a \frac{1}{n} \sum_{k=1}^{n} u_{k}^{(1,2,3)}=a T u,
$$

that is, $T$ has the property of homogeneity. Thus, $T$ is linear. Let us take any $v \in \ell_{\infty}\left(F_{t}\right)$ and represent the sequence $u=\left(u_{k}\right)$ using the $\mathscr{C}^{-1}$ as $u=$ $\left(u_{k}^{(1,2,3)}\right)=\left(\mathfrak{B}^{k} v_{j}^{(1,2,3)}\right)$ where $\mathfrak{B}^{k}=\sum_{j=0}^{k}(-1)^{n-j} j$. Then, we have

$$
\|u\|_{\left[\ell_{\infty}\left(F_{t}\right)\right]_{\mathscr{C}}}=\sup _{k \in \mathbb{N}} \bar{d}\left(\mathscr{C} u_{k}^{(1,2,3)}, \overline{0}\right)=\sup _{k \in \mathbb{N}} \bar{d}\left(\frac{1}{n} \sum_{k=1}^{n}\left[\mathfrak{B}^{k} v_{j}^{(1,2,3)}\right], \overline{0}\right)=\sup _{k \in \mathbb{N}} \bar{d}\left(v_{j}^{(1,2,3)}, \overline{0}\right)=\|v\|_{\ell_{\infty}\left(F_{t}\right)} .
$$

Namely, $T$ is norm preserving. Consequently, the spaces $\left[\ell_{\infty}\left(F_{t}\right)\right]_{\mathscr{C}}$ and $\ell_{\infty}\left(F_{t}\right)$ are linearly isomorphic. It is clear here that if the spaces $\left[\ell_{\infty}\left(F_{t}\right)\right]_{\mathscr{C}}$ and $\ell_{\infty}\left(F_{t}\right)$ are replaced by the spaces $\left[c\left(F_{t}\right)\right]_{\mathscr{C}}$ and $c\left(F_{t}\right),\left[c_{0}\left(F_{t}\right)\right]_{\mathscr{C}}$ and $c_{0}\left(F_{t}\right)$, then we obtain the fact that $\left[c\left(F_{t}\right)\right]_{\mathscr{C}} \cong c\left(F_{t}\right)$ and $\left[c_{0}\left(F_{t}\right)\right]_{\mathscr{C}} \cong c_{0}\left(F_{t}\right)$. This completes the proof. 
Theorem 3.2 The sets $\left[c\left(F_{t}\right)\right]_{\mathscr{C}},\left[c_{0}\left(F_{t}\right)\right]_{\mathscr{C}}$ and $\left[\ell_{\infty}\left(F_{t}\right)\right]_{\mathscr{C}}$ are complete module sequence space of the fuzzy numbers with the module defined by

$$
\|u\|=\sup _{k} \max _{i=1,2,3}\left|\mathscr{C} u^{(i)}\right| .
$$

Proof: As was shown in Theorem 3.1, the sequence spaces of triangular fuzzy numbers $\left[\ell_{\infty}\left(F_{t}\right)\right]_{\mathscr{C}},\left[c\left(F_{t}\right)\right]_{\mathscr{C}}$ and $\left[c_{0}\left(F_{t}\right)\right]_{\mathscr{C}}$ are linearly isomorphic to the spaces $\ell_{\infty}\left(F_{t}\right), c\left(F_{t}\right)$ and $c_{0}\left(F_{t}\right)$, respectively. In addition to this, since the matrix $\mathscr{C}$ is normal (see, [11]) and $\ell_{\infty}\left(F_{t}\right), c\left(F_{t}\right)$ and $c_{0}\left(F_{t}\right)$ are complete module spaces $[9]$, it is clear that the sequence spaces $\left[\ell_{\infty}\left(F_{t}\right)\right]_{\mathscr{C}},\left[c\left(F_{t}\right)\right]_{\mathscr{C}}$ and $\left[c_{0}\left(F_{t}\right)\right]_{\mathscr{C}}$ are complete module spaces with the module defined in (11).

Theorem 3.3 Let $\left(u_{k}\right)=\left(u_{k}^{(1,2,3)}\right)$ be a sequence of fuzzy numbers. If $\bar{d}\left(u_{k}^{(1,2,3)}, u_{0}^{(1,2,3)}\right) \rightarrow 0$ as $n \rightarrow \infty$ then $\bar{d}\left(\frac{1}{n} \sum_{k=1}^{n} u_{k}^{(1,2,3)}, u_{0}^{(1,2,3)}\right) \rightarrow 0$ as $n \rightarrow \infty$. It means that the matrix $\mathscr{C}$ is regular.

Proof: Let $\left(u_{k}\right)=\left(u_{k}^{(1,2,3)}\right)$ be a sequence of triangular fuzzy numbers and $\bar{d}\left(u_{k}^{(1,2,3)}, u_{0}^{(1,2,3)}\right) \rightarrow 0$ as $n \rightarrow \infty$. Then for a given $\epsilon>0$ there exist a positive integer $n_{0}$ such that

$$
\bar{d}\left(u_{k}^{(1,2,3)}, u_{0}^{(1,2,3)}\right)<\epsilon \text { for } n \geq n_{0} .
$$

Therefore, we can write easily by using the arithmetic mean property of limit function as follows:

$$
\bar{d}\left(\mathscr{C} u_{k}^{(1,2,3)}, u_{0}^{(1,2,3)}\right)=\bar{d}\left(\frac{1}{n} \sum_{k=1}^{n} u_{k}^{(1,2,3)}, u_{0}^{(1,2,3)}\right)<\epsilon \quad \text { for } n \geq n_{0}, n_{0} \in \mathbb{N} .
$$

This step completes the proof.

Theorem 3.4 The inclusions $c\left(F_{t}\right) \subset\left[c\left(F_{t}\right)\right]_{\mathscr{C}}$ and $c_{0}\left(F_{t}\right) \subset\left[c_{0}\left(F_{t}\right)\right]_{\mathscr{C}}$ strictly hold.

Proof: To prove the validity of the inclusion $c_{0}\left(F_{t}\right) \subset\left[c_{0}\left(F_{t}\right)\right]_{\mathscr{C}}$, let us take any $v \in c_{0}\left(F_{t}\right)$. Then, bearing in mind the regularity of the method $\mathscr{C}$ (see, Theorem 3.3), we immediately observe that $\mathscr{C} v \in c_{0}\left(F_{t}\right)$ which means that $v \in\left[c_{0}\left(F_{t}\right)\right]_{\mathscr{C}}$. Hence, the inclusion $c_{0}\left(F_{t}\right) \subset\left[c_{0}\left(F_{t}\right)\right]_{\mathscr{C}}$ holds. Furthermore, let us consider the sequence $u=\left(u_{k}^{(1,2,3)}\right)$ defined by $u_{k}^{(1,2,3)}=(-1)^{k}\left(\frac{k-1}{k}, \frac{3}{2}, \frac{3 k-1}{k}\right)$ for all $k \in \mathbb{N}$. Then, we have $\left(\mathscr{C} u_{k}^{(1,2,3)}\right)=\frac{1}{k} \begin{cases}(-k, 0, k), & \mathrm{k} \text { is odd; } \\ -\left(k+1, \frac{-3}{2}, k-1\right), & \mathrm{k} \text { is even, }\end{cases}$ which implies that $u$ is in $\left[c_{0}\left(F_{t}\right)\right]_{\mathscr{C}}$ but $u \notin c_{0}\left(F_{t}\right)$. This shows that the inclusion is strict. One can see by analogously that the strict inclusion $c\left(F_{t}\right) \subset$ $\left[c\left(F_{t}\right)\right]_{\mathscr{C}}$ also holds. This completes the proof. 
Let $\lambda(F), \mu(F) \subset w(F)$ and $\mathscr{A}=\left(a_{n k}\right)$ be an infinite matrix of fuzzy numbers and consider following expressions:

$$
\begin{array}{r}
\sup _{n \in \mathbb{N}} \sum_{k} \bar{d}\left(a_{n k}, \overline{0}\right)<\infty, \\
\sup _{n \in \mathbb{N}} \sum_{k}\left[\bar{d}\left(a_{n k}, \overline{0}\right)\right]^{q}<\infty, \\
\lim _{n} \bar{d}\left(a_{n k}, \alpha_{k}\right)=0, \quad \text { where } \alpha_{k} \in w(F), \\
\lim _{n} \sum_{k} \bar{d}\left(a_{n k}, \overline{0}\right)=0, \\
\lim _{n} \sum_{k} a_{n k}=\overline{1}, \\
\lim _{n} a_{n k}=\overline{0}, \quad k \in \mathbb{N} .
\end{array}
$$

In [10], some matrix classes are characterized by Talo and Başar which is given in the following lemma:

Lemma 3.5 [10] The following statements hold:

(18) $\mathscr{A} \in\left(\ell_{\infty}\left(F_{t}\right): \ell_{\infty}\left(F_{t}\right)\right), \mathscr{A} \in\left(c\left(F_{t}\right): \ell_{\infty}\left(F_{t}\right)\right), \mathscr{A} \in\left(c_{0}\left(F_{t}\right): \ell_{\infty}\left(F_{t}\right)\right)$ if and only if (13) holds,

(19) $\mathscr{A} \in\left(\ell_{\infty}\left(F_{t}\right): c_{0}\left(F_{t}\right)\right)$ if and only if (16) holds,

(20) $\mathscr{A} \in\left(c_{0}\left(F_{t}\right): c\left(F_{t}\right)\right)$ if and only if (13) and (15) hold,

(21) $\mathscr{A} \in\left(c_{0}\left(F_{t}\right): c_{0}\left(F_{t}\right)\right)$ if and only if (13) and (15) hold with $a_{k}=\overline{0}$, $\forall k \in \mathbb{N}$,

(22) $\mathscr{A} \in\left(\ell_{p}\left(F_{t}\right): c\left(F_{t}\right)\right)$ if and only if (14) and (15) hold,

(23) $\mathscr{A} \in\left(\ell_{p}\left(F_{t}\right): c_{0}\left(F_{t}\right)\right)$ if and only if (14) and (15) hold with $a_{k}=\overline{0}$, $\forall k \in \mathbb{N}$,

(24) $\mathscr{A} \in\left(c\left(F_{t}\right): c\left(F_{t}\right), p\right)$ if and only if (13), (17) and (15) hold with $a_{k}=\overline{0}$, $\forall k \in \mathbb{N}$.

\section{Real Duals of the spaces $\left[\ell_{\infty}\left(F_{t}\right)\right]_{\mathscr{C}},\left[c\left(F_{t}\right)\right]_{\mathscr{C}}$ and $\left[c_{0}\left(F_{t}\right)\right]_{\mathscr{C}}$}

In this section, we state and prove the theorems determining the $\alpha(r)$-, $\beta(r)$ and $\gamma(r)$ - real duals of the spaces $\left[\ell_{\infty}\left(F_{t}\right)\right]_{\mathscr{C}},\left[c\left(F_{t}\right)\right]_{\mathscr{C}}$ and $\left[c_{0}\left(F_{t}\right)\right]_{\mathscr{C}}$. For the sequence spaces $\lambda(F)$ and $\mu(F)$, define the set

$$
S(\lambda(F), \mu(F))=\left\{a^{(1,1,1)} \in w(\mathbb{R}):\left(a_{k} x_{k}^{(1,2,3)}\right) \in \mu(F) \text { for all } x \in \lambda(F)\right\},
$$

where $w(\mathbb{R})$ denotes all real valued sequences space. Then, $\alpha(r)-, \beta(r)$ - and $\gamma(r)$ - duals of a sequence space $\lambda(F)$ are defined by $\lambda^{\alpha(r)}(F)=S\left(\lambda(F), \ell_{1}(F)\right), \quad \lambda^{\beta(r)}(F)=$ $S(\lambda(F), c s(F))$ and $\lambda^{\gamma(r)}(F)=S(\lambda(F), b s(F))$, respectively. 
Theorem 4.1 The $\gamma(r)$-dual of the spaces $\left[\ell_{\infty}\left(F_{t}\right)\right]_{\mathscr{C}},\left[c\left(F_{t}\right)\right]_{\mathscr{C}}$ and $\left[c_{0}\left(F_{t}\right)\right]_{\mathscr{C}}$ is the set

$$
D_{1}=\left\{a \in w(\mathbb{R}): \sup _{n \in \mathbb{N}} \sum_{k=0}^{n} \bar{d}\left(\sum_{j=k}^{n}(-1)^{j-k} k a_{j}, \overline{0}\right)<\infty\right\} .
$$

Proof: Since the proof is similar for the rest of the spaces, we determine only $\gamma(r)$ - dual of the set $\left[c_{0}\left(F_{t}\right)\right]_{\mathscr{C}}$. Let $a^{(1,1,1)} \in w(\mathbb{R})$ and define the matrix $\mathscr{Z}=\left(z_{n k}\right)$ via the sequence $a=\left(a_{i}^{(1,1,1)}\right)$ by

$$
z_{n k}=\left\{\begin{array}{ll}
\sum_{j=k}^{n}(-1)^{j-k} k a_{j} & , \quad(0 \leq k \leq n) \\
0 \quad, \quad(k>n)
\end{array} ;(k, j, n \in \mathbb{N})\right.
$$

Bearing in mind the relation (10) we immediately derive that

$$
\sum_{k=0}^{n} a_{k}^{(1,1,1)} x_{k}^{(1,2,3)}=\sum_{k=0}^{n}\left[\sum_{j=k}^{n}(-1)^{j-k} k a_{j} y_{k}\right]=\left(\mathscr{Z} y^{(1,2,3)}\right)_{n} \quad, \quad(k, j, n \in \mathbb{N}) .(21)
$$

From $(21)$ we realize that $a x=\left(a_{k}^{(1,1,1)} x_{k}^{(1,2,3)}\right) \in b s\left(F_{t}\right)$ whenever $x \in\left[c_{0}\left(F_{t}\right)\right]_{\mathscr{C}}$ if and only if $\mathscr{Z} y^{(1,2,3)} \in \ell_{\infty}\left(F_{t}\right)$ whenever $y^{(1,2,3)} \in c_{0}\left(F_{t}\right)$. Then, by considering the Part (18) of Lemma 3.5, we have $\sup _{n \in \mathbb{N}} \sum_{k=0}^{n} \bar{d}\left(\sum_{j=k}^{n}(-1)^{j-k} k a_{j}^{(1,1,1)}, \overline{0}\right)<$ $\infty$ which yields the consequence that $\left[c_{0}\left(F_{t}\right)\right]_{\mathscr{C}}^{\gamma(r)}=D_{1}$.

Theorem $4.2\left[c_{0}\left(F_{t}\right)\right]_{\mathscr{C}}^{\alpha(r)}$ is the set

$$
D_{2}=\left\{a \in w(\mathbb{R}): \sup _{\mathbb{N}} \sum_{k} \bar{d}\left(\sum_{n \in \mathbb{N}} \sum_{j=k}^{n}(-1)^{j-k} k a_{j}^{(1,1,1)}, \overline{0}\right)<\infty\right\} .
$$

Proof: The proof is similar to the proof of Theorem 4.1.

Lemma 4.3 ([1], Theorem 3.1) Let $\mathscr{W}=\left(w_{n k}\right)$ be defined via a sequence $a=\left(a_{k}\right) \in w$ and the inverse matrix $\mathscr{V}=\left(v_{n k}\right)$ of the triangle matrix $\mathscr{U}=$ $\left(u_{n k}\right)$ by $w_{n k}=\left\{\begin{array}{lc}\sum_{j=k}^{n} a_{j} v_{j k}, & 0 \leq k \leq n, \\ 0, & k>n,\end{array}\right.$ for all $k, n \in \mathbb{N}$. Then $\left\{\lambda_{\mathscr{U}}\right\}^{\gamma}=$ $\left\{a=\left(a_{k}\right) \in w: \mathscr{C} \in\left(\lambda: \ell_{\infty}\right)\right\}$ and $\left\{\lambda_{\mathscr{U}}\right\}^{\beta}=\left\{a=\left(a_{k}\right) \in w: \mathscr{C} \in(\lambda: c)\right\}$.

Theorem 4.4 Define the sets $D_{3}, D_{4}$ and $D_{5}$ as in the following:

$$
\begin{gathered}
D_{3}=\left\{a \in w(\mathbb{R}): \lim _{n \rightarrow \infty} \bar{d}\left(\sum_{j=k}^{n}(-1)^{j-k} k a_{j}^{(1,1,1)}, \alpha_{k}\right)=0, \alpha_{k} \in w(F)\right\}, \\
D_{4}=\left\{a \in w(\mathbb{R}): \lim _{n \rightarrow \infty} \bar{d}\left(\sum_{j=k}^{n}(-1)^{j-k} k a_{j}^{(1,1,1)}, \overline{0}\right) \text { exists }\right\}
\end{gathered}
$$




$$
D_{5}=\left\{a \in w(\mathbb{R}): \lim _{n \rightarrow \infty} \sum_{k=0}^{n}\left(\sum_{j=k}^{n}(-1)^{j-k} k a^{(1,1,1)}\right)=\overline{1}\right\} .
$$

Then, $\left[c_{0}\left(F_{t}\right)\right]_{\mathscr{C}}^{\beta(r)}=D_{1} \cap D_{3}$. And $\left[c\left(F_{t}\right)\right]_{\mathscr{C}}^{\beta(r)}=\cup_{i=4}^{5} D_{i} \cup D_{1}$.

Proof: The proof is clear from Lemma 4.3.

\section{Matrix Transformations}

Let us suppose that the set $\left[\lambda\left(F_{t}\right)\right]_{\mathscr{C}}$ be any of the sets $\left[c_{0}\left(F_{t}\right)\right]_{\mathscr{C}},\left[c\left(F_{t}\right)\right]_{\mathscr{C}}$ and $\left[\ell_{\infty}\left(F_{t}\right)\right]_{\mathscr{C}}$. In this section, we characterize the matrix mappings from $\left[\lambda\left(F_{t}\right)\right]_{\mathscr{C}}$ into any given sequence space of fuzzy numbers via the concept of the dual summability methods and vice versa, so we call it as the Cesàro dual summability methods on the sequence of fuzzy numbers. Let us suppose that the sequences $u=\left(u_{i}^{(1,2,3)}\right)$ and $v=\left(v_{i}^{(1,2,3)}\right)$ are connected with $(10)$ and let the $\mathscr{A}$ - transform of the sequence $u$ be $z=\left(z_{i}^{(1,2,3)}\right)$ and the $\mathscr{B}$-transform of the sequence $v$ be $t=\left(t_{i}\right)$, i.e.,

$$
\begin{aligned}
& z_{i}^{(1,2,3)}=\left(\mathscr{A} u^{(1,2,3)}\right)_{i}=\sum_{i} a_{n i}^{(1,1,1)} u_{i}^{(1,2,3)}, \quad(i \in \mathbb{N}) \\
& t_{i}^{(1,2,3)}=\left(\mathscr{B} v^{(1,2,3)}\right)_{i}=\sum_{i} b_{n i}^{(1,1,1)} v_{i}^{(1,2,3)}, \quad(i \in \mathbb{N}) .
\end{aligned}
$$

It is clear here that the method $\mathscr{B}$ is applied to the $\mathscr{C}$ - transform $v=$ $\left(v_{i}^{(1,2,3)}\right)=\left\{(\mathscr{C} u)_{i}\right\}$ of the sequence $u=\left(u_{i}^{(1,2,3)}\right)$ while the method $\mathscr{A}$ is directly applied to the terms of the sequence $u=\left(u_{i}^{(1,2,3)}\right)$. So, the methods $\mathscr{A}$ and $\mathscr{B}$ are essentially different (see, [2]).

Let us assume the existence of the matrix product $\mathscr{B} \mathscr{C}$ which is a much weaker assumption than the conditions on the matrix $\mathscr{B}$ belonging to any matrix class, in general. If $z_{i}^{(1,2,3)}$ becomes $t_{i}^{(1,2,3)}$ under the application of the formal summation by parts, then the methods $\mathscr{A}$ and $\mathscr{B}$ are called Cesàro dual type matrices. This leads us to fact that $\mathscr{B} \mathscr{C}$ exists and is equal to $\mathscr{A}$ and $\mathscr{A}=(\mathscr{B} \mathscr{C}) u^{(1,2,3)}=\mathscr{B}\left(\mathscr{C} u^{(1,2,3)}\right)=\mathscr{B} v$ formally holds. This statement is equivalent to the following relation between the elements of the matrices $\mathscr{A}=\left(a_{n i}\right)$ and $\mathscr{B}=\left(b_{n i}\right)$,

$$
b_{n i}^{(1,1,1)}=\sum_{i=1}^{n}(-1)^{n-i} i a_{n i}^{(1,1,1)} \quad \text { or } \quad a_{n i}^{(1,1,1)}=\frac{1}{n} \sum_{i=1}^{n} b_{n i}^{(1,1,1)}
$$


for all $n, i \in \mathbb{N}$. Furthermore one can easily see that $v_{i}^{(1,2,3)}$ reduces $u_{i}^{(1,2,3)}$, as in the following,

$$
v_{i}^{(1,2,3)}=\sum_{i} b_{n i} y_{i}^{(1,2,3)}=\sum_{i} b_{n i}\left(\sum_{j=0}^{i} x_{j}^{(1,2,3)}\right)=\sum_{j} \sum_{i=j}^{\infty} b_{n i} x_{j}^{(1,2,3)}=u_{i}^{(1,2,3)}
$$

Now we may give the following theorem concerning to the Cesàro dual matrices:

Theorem 5.1 Let $\mathscr{A}=\left(a_{n i}^{(1,1,1)}\right)$ and $\mathscr{B}=\left(b_{n i}^{(1,1,1)}\right)$ be the Cesàro dual summability methods on the sequence of fuzzy numbers, $\mu(F)$ be any given sequence space of the fuzzy numbers and $\left(a_{n i}^{(1,1,1)}\right)_{i \in \mathbb{N}} \in \ell_{1}\left(F_{t}\right)$. Then, $\mathscr{A} \in$ $\left(\left[c\left(F_{t}\right)\right]_{\mathscr{C}}: \mu(F)\right)$ if and only if $\mathscr{B} \in\left(c\left(F_{t}\right): \mu(F)\right)$.

Proof: Suppose that $\mathscr{A}=\left(a_{n i}^{(1,1,1)}\right)$ and $\mathscr{B}=\left(b_{n i}^{(1,1,1)}\right)$ be Cesàro dual type summability methods on the sequence of the fuzzy numbers which means that (27) holds. In addition to this, let $\mu(F)$ be any given sequence space and take account that the spaces $\left[c\left(F_{t}\right)\right]_{\mathscr{C}}$ and $c\left(F_{t}\right)$ are linearly isomorphic.

Let $\mathscr{A} \in\left(\left[c\left(F_{t}\right)\right]_{\mathscr{C}}: \mu(F)\right)$ and take any $y \in c\left(F_{t}\right)$. Then, $\mathscr{B} \mathscr{C}$ is equal to $\mathscr{A}$ and $\left(a_{n i}^{(1,1,1)}\right)_{i \in \mathbb{N}} \in\left[c\left(F_{t}\right)\right]_{\mathscr{C}}^{\beta(r)}$ which yields that $\left(b_{n i}^{(1,1,1)}\right)_{i \in \mathbb{N}} \in \ell_{1}\left(F_{t}\right)$ for each $n \in \mathbb{N}$. Hence $\mathscr{B} y^{(1,2,3)}$ exists for each $y \in c\left(F_{t}\right)$ and we have the following equation,

$$
\sum_{i} b_{n i}^{(1,1,1)} y_{i}^{(1,2,3)}=\sum_{i} a_{n i}^{(1,1,1)} x_{i}^{(1,2,3)} \quad(n \in \mathbb{N})
$$

Subsequently, it is clear from $(27)$ that $\mathscr{B} y=\mathscr{A} x$ which leads us to the consequence $\mathscr{B} \in\left(c\left(F_{t}\right): \mu(F)\right)$. Conversely, suppose $\mathscr{B} \in\left(\left[c\left(F_{t}\right)\right]_{\mathscr{C}}: \mu(F)\right)$ and take any $x \in\left[c\left(F_{t}\right)\right]_{\mathscr{C}}$. Then, $\mathscr{A} x$ exists. Therefore, we obtain from the following equality as $n \rightarrow \infty$ that $\mathscr{A} x=\mathscr{B} y$ and this shows that $\mathscr{A} \in\left(\left[c\left(F_{t}\right)\right]_{\mathscr{C}}: \mu(F)\right)$ under the equation $\sum_{i=0}^{n} a_{n i}^{(1,1,1)} x_{i}^{(1,2,3)}=\sum_{i=0}^{n}\left\{\sum_{i=0}^{n}(-1)^{n-i} i a_{n i}^{(1,1,1)}\right\} y_{i} ; \quad(n \in$ $\mathbb{N}$ ). This step completes the proof.

Theorem 5.2 Suppose that the elements of the infinite matrices $\mathscr{D}=\left(d_{n k}^{(1,1,1)}\right)$ and $\mathscr{E}=\left(e_{n k}^{(1,1,1)}\right)$ are connected with the relation

$$
e_{n k}^{(1,1,1)}=\frac{1}{n} \sum_{j=0}^{n} d_{j k}^{(1,1,1)}, \quad(k, n \in \mathbb{N})
$$

and $\mu(F)$ be any given sequence space. Then, $\mathscr{D} \in\left(\mu(F):\left[c\left(F_{t}\right)\right]_{\mathscr{C}}\right)$ if and if only $\mathscr{E} \in\left(\mu(F): c\left(F_{t}\right)\right)$. 
Proof: Let $x=\left(x_{k}\right) \in \mu(F)$ and consider the following equality with (30),

$$
\{\mathscr{C}(\mathscr{D} x)\}_{n}=\frac{1}{i} \sum_{j=0}^{i} \sum_{k=0}^{n} d_{j k}^{(1,1,1)} x_{k}^{(1,2,3)}=\sum_{k=0}^{n} e_{i k}^{(1,1,1)} x_{k}^{(1,2,3)}, \forall i, n \in \mathbb{N}
$$

which yields as $n \rightarrow \infty$ that $\frac{1}{i} \sum_{j=0}^{i}\left(\mathscr{D} x^{(1,2,3)}\right)_{j}=\left(\mathscr{E} x^{(1,2,3)}\right)_{i}, \forall i \in \mathbb{N}$. From here we obtain that $\mathscr{D} x^{(1,2,3)} \in\left[c\left(F_{t}\right)\right]_{\mathscr{C}}$ whenever $x \in \mu(F)$. This completes the proof.

\section{References}

[1] B. Altay, F. Başar, Certain topological properties and duals of the matrix domain of a triangle matrix in a sequence space, J. Math. Anal. Appl., Vol.336,1(2007), 632-645. http://dx.doi.org/10.1016/j.jmaa.2007.03.007

[2] F. Başar, Matrix transformations between certain sequence spaces of $X_{p}$ and $\ell_{p}$, Soochow J. Math., Vol.26, 2(2000), 191-204.

[3] D. Filev and R. Yager, A generalized defuzzification method under BAD distributions, Int. J. Intell. Syst., 6(1991), 689-697.

[4] Z. Mitrovic and S. Rusov, Z Similarity measure among fuzzy sets, FME Transactions, 34(2006), 115-119.

[5] R. E. Moore, Automatic error analysis in digital computation, Tech. Rep., LSMD- 48421, Lockheed Missiles and Space Company, (1959).

[6] M. L. Puri and D. A. Ralescu, Differentials for fuzzy functions, J. Math. Anal. Appl., 91(1983), 552-558. http://dx.doi.org/10.1016/0022$247 x(83) 90169-5$

[7] M. Sugeno, An introductory survey of fuzzy control, Inf. Sci., Vol.36, 1-2(1985), 59-83. http://dx.doi.org/10.1016/0020-0255(85)90026-x

[8] M. Şengönül and F. Başar, Some new Cesàro sequence spaces of nonabsolute type which include the spaces $c_{0}$ and $c$, Soochow J. Math., Vol.31, $1(2005), 107-119$.

[9] M. Şengönül, On the Zweier sequence spaces of fuzzy numbers, Int. J. Modern Math. Sci., 439169, 2014. http://dx.doi.org/10.1155/2014/439169 
[10] Ö. Talo and F. Başar, Determination of the duals of classical sets of sequences of fuzzy numbers and related matrix transformations, Comput. Math. Appl., Vol.58, 4(2009), 717-733. http://dx.doi.org/10.1016/j.camwa.2009.05.002

[11] A. Wilansky, Summability through functional analysis, North-Holland Mathematics Studies 85, Elsevier Science Publishers, Amsterdam, 1984.

[12] L. A. Zadeh, Fuzzy sets, Inf. Control, 8(1965), 338-353. http://dx.doi.org/10.1016/s0019-9958(65)90241-x

[13] Z. Zararsız and M. Şengönül, On the gravity of center of sequence of fuzzy numbers, 6(2013).

Received: November 20, 2014; Published: January 3, 2015 